\title{
AN INTEGRAL INEQUALITY FOR $n$-CONVEX FUNCTIONS
}

\section{HACÈne Belbachir AND Mourad RAHMANi}

Abstract. We extend Lupaş inequality for $n$-convex ( $n$-concave) functions. As consequences some inequalities are derived.

Mathematics subject classification (2010): 26D15, 26D10, 33C45.

Keywords and phrases: Čebyšev functional, convex functions of higher order, Legendre polynomials.

\section{REFERENCES}

[1] G. E. Andrews, R. Askey And R. Roy, Special Functions, Cambridge University Press, Cambridge, 1999.

[2] E. V. AtKinson, An Inequality, Univ Beograd. Publ. Elektrotehn. Fak. Ser. Mat. Fiz. 357-380 (1971), $5-6$.

[3] V. Сiobotariu-Boer, An Integral Inequality for 3-convex Functions, J. Inequl. Pur and Appl. Math. 9, 4 (2008), Art. 98

[4] C. Krattenthaler, Advanced determinant calculus, Sém. Lothar. Combin. 42 (1999), Art. B42q, $67 \mathrm{pp}$.

[5] M. Kuczma, An Introduction to the Theory of Functional Equtions and Inequlities. Cauchy's Equations and Jensen's Inequalities, Birkhäuser Verlag AG, 2nd Ed, 2009.

[6] A. LuPAŞ, An Integral Inequality for Convex Functions, Univ Beograd. Publ. Elektrotehn. Fak. Ser. Mat. Fiz. 381-409 (1972), 17-19.

[7] D. S. Mitrinović, J. E. PeČArić And A. M. FinK, Classical and New Inequalities in Analysis, Kluwer Academic Publishers, 1993.

[8] J. E. PEČARIĆ, On an Integral Inequality of A. Lupaş, Rev. Anal. Numer. Theor. Approx. 23, 1 (1994), $101-102$.

[9] J. E. PeČarić, F. Proschan and Y. L. Tong, Convex Functions, Partial Orderings, and Statistiacl Applications, Academic Press, San Diego, 1992.

[10] G. SzEGÖ, Orthogonal Polynomials, Colloquium Publications, vol. 23, Amer. Math. Soc., Providence, 1975. 